\title{
Nonlinear Tamm states in nanostructured plasmonic metamaterials
}

\author{
Ivan V. Iorsh, ${ }^{1,2}$ Pavel A. Belov, ${ }^{1,3}$ Alexander A. Zharov, ${ }^{4}$ Ilya V. Shadrivov, ${ }^{1,5}$ and Yuri S. Kivshar ${ }^{1,5}$ \\ ${ }^{1}$ National Research University of Information Technologies, Mechanics and Optics (ITMO), St. Petersburg 197101, Russia \\ ${ }^{2}$ Department of Physics, Durham University, DH1 3LE, Durham, United Kingdom \\ ${ }^{3}$ Electronic Engineering, Queen Mary University of London, Mile End Road, London E1 4NS, United Kingdom \\ ${ }^{4}$ Institute for Physics of Microstructures, Russian Academy of Sciences, Nizhny Novgorod 603950, Russia \\ ${ }^{5}$ Nonlinear Physics Centre, Research School of Physics and Engineering, Australian National University, Canberra ACT 0200, Australia
}

(Received 4 May 2012; published 10 August 2012)

\begin{abstract}
We analyze nonlinear surface modes supported by a metal-dielectric nanostructured metamaterial with a nonlinear surface layer. We demonstrate that such a semi-infinite structure can support both TE and TM polarized surface modes with the subwavelength localization, which can be regarded as an optical analog of the electronic Tamm states. Such nonlinear Tamm states may appear even in the cases when linear surface modes do not exist.
\end{abstract}

DOI: 10.1103/PhysRevA.86.023819

PACS number(s): 42.25.Gy

\section{INTRODUCTION}

Surface modes appear in different problems of physics as a special type of mode localized near an interface separating two different media. In optics, linear electromagnetic surface waves are known to exist on metal surfaces at an interface separating homogeneous and periodic dielectric media [1] as well as in layered structures containing thin metal films [2-4].

The interest in the study of nonlinear electromagnetic surface waves has been renewed recently, and it was shown theoretically [5-7] and experimentally [8-11] that nonlinearityinduced self-trapping of light may become possible near the edge of a one-dimensional waveguide array leading to the formation of nonlinear Tamm states (see also [12], the review [13], and references therein). In particular, it was found that the surface modes acquire some novel properties in the nonlinear regime. Such self-trapped nonlinear waves exist above a certain threshold power, and they may demonstrate bistability when for the same value of the mode power two different surface modes coexist.

Recently, it was shown that strongly confined plasmonic surface modes can exist at the termination of metal-dielectric metamaterials [14-16]. It is required to employ binary structures to reduce the lattice symmetry in order to support surface waves [16]. Such localized modes demonstrate many properties that can be useful in photonic or plasmonic sources. Indeed, due to their specific dispersion and hybrid metaldielectric nature of the structures that support such surface modes, they allow coupling either to the optical mode inside the light cone or directly to the plasmon mode. More importantly, such surface modes are associated with lower losses than conventional plasmons, and they can be controlled and laterally confined by a simple patterning of the metal layer. In addition, the surface metallic layer can also allow a simple electrical injection scheme for the realization of plasmonic or photonic integrated sources.

In this paper we demonstrate that the terminated metaldielectric nanostructured metamaterials, the so-called plasmonic metamaterials, can support a variety of TE and TM polarized surface modes in the nonlinear regime, and we analyze the properties of the nonlinear Tamm states in such structures, including their stability. We also demonstrate how to generate both linear and nonlinear surface modes by an incoming electromagnetic radiation.
The paper is organized as follows. In Sec. II A we discuss the TE polarized nonlinear surface modes which generalize the familiar linear surface modes, but may acquire some unusual properties. Section II B is devoted to the study of the TM polarized surface modes in both linear and nonlinear regimes. In Sec. III we demonstrate, for the simpler example of the TE polarized modes, how to generate nonlinear surface modes by incoming radiation coupled to the periodic structure. Finally, Sec. IV concludes the paper.

\section{NONLINEAR SURFACE MODES}

Two possible geometries of the terminated metal-dielectric structures under consideration are shown in Figs. 1(a) and 1(b) where we assume that all layers have subwavelength thickness. In what follows, we consider separately the cases of TE and TM polarizations.

\section{A. TE polarized modes}

For the case of TE-polarized waves and isotropic nonsaturable Kerr nonlinearity, dielectric permittivity of a thin nonlinear layer can be written as

$$
\varepsilon=\varepsilon_{\text {lin }}+\chi_{3}\left|E_{y}\right|^{2} .
$$

We look for the electric and magnetic fields in the following form: $\left(\mathcal{E}_{y}, \mathcal{H}_{x}\right) \sim e^{i(\beta z-\omega t)}$. In this case, Maxwell's equations read

$$
H_{z}=\frac{i}{k_{0}} \frac{\partial E_{y}}{\partial x}, \quad H_{x}=\frac{\beta}{k_{0}} E_{y}, \quad \frac{\partial H_{z}}{\partial x}=i \beta H_{x}-i k_{0} \varepsilon E_{y},
$$

where $k_{0}=\omega / c$. From Eqs. (2) we derive the nonlinear differential equation for the field amplitude:

$$
\frac{\partial^{2} E_{y}}{\partial x^{2}}+\left(\varepsilon k_{0}^{2}-\beta^{2}\right) E_{y}=0 .
$$

Now we take into account that the nonlinear layer is thin. We assume that the nonlinear permittivity is constant across the layer, and the nonlinearity is weak: $\delta \varepsilon_{\mathrm{nl}} / \varepsilon \ll 1$. In this case, we write down the formal solution of Eq. (3) in the thin layer as

$$
E_{y}=A \sin [q(x+d)]+B \cos [q(x+d)],
$$




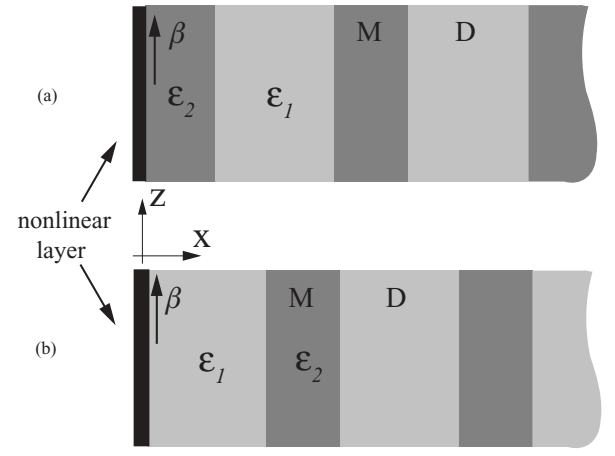

FIG. 1. Geometries of the structure under consideration: (a) metal layer terminates the structure; (b) dielectric layer terminates the structure.

where $q=\sqrt{\varepsilon k_{0}^{2}-\beta^{2}}$, and $d$ is the thickness of the nonlinear layer. We then formulate the continuity boundary conditions at the layer interfaces. For this, it is convenient to introduce a surface admittance for the metal-dielectric nanostructure: $Y_{T E}=H_{z} / E_{y}$. Surface admittance can be expressed through the components of the transfer matrix for the period of the metal-dielectric nanostructure:

$$
Y_{T E}=\frac{e^{i K D}-T_{11}^{T E}}{T_{12}^{T E}},
$$

where $K$ and $D$ are the Bloch wave vector and period of the structure, respectively. Expressions for the components of the transfer matrices are textbook values, and they can be found, e.g., in Ref. [1].

Field outside the structure should decay exponentially, so it is convenient to write the field amplitude as $E_{y} \sim$ $\exp [\kappa(x+d)]$, where $\kappa=\sqrt{\beta^{2}-k_{0}^{2}}$. If we then exclude all the amplitudes using boundary conditions, we end up with the following equation:

$$
\left(\frac{\kappa}{k_{0}}+i Y_{T E}\right)=k_{0} d\left(\varepsilon-\frac{\beta^{2}}{k_{0}^{2}}-i Y_{T E} \frac{\kappa}{k_{0}}\right) .
$$

We note that if we set the right-hand side of Eq. (6) to zero, we obtain the dispersion of the surface states without the nonlinear layer. These equations are somewhat analogous to the boundary conditions for thin layers widely used in microwave theory (see, e.g., Ref. [17]).

A nonlinear correction to the dielectric permittivity $\varepsilon$ reads

$$
\varepsilon-\varepsilon_{\text {lin }}=\chi_{3}\left|E_{y}\right|^{2}=\chi_{3} I_{0},
$$

where $I_{0}$ is the wave intensity at the nonlinear layer boundary. The sign of $\chi_{3}$ defines the type of nonlinearity: positive $\chi_{3}$ corresponds to focusing nonlinearity, while negative corresponds to defocusing nonlinearity. We consider focusing nonlinearity, and the field amplitude normalized such that $\chi_{3}=1$. As a result, we derive the expressions relating the propagation constant $\beta$, frequency $\omega$, and field intensity $I_{0}$. If we fix the frequency and change $\beta$ then for each value of the propagation constant we obtain the corresponding field intensity. If the field intensity vanishes then the propagation constant and frequency usually correspond to the linear solution.

To calculate the energy flow, we integrate the Poynting vector across the structure. For the TE polarization, the energy flow reads

$$
\begin{aligned}
\mathrm{P} & =\int_{-\infty}^{\infty} d x S_{z}=\frac{1}{2} \int_{-\infty}^{\infty} \operatorname{Re}\left(E_{y} H_{x}^{*}\right) d x \\
& =\frac{\beta}{2 k_{0}}\left|E_{y}(0)\right|^{2} \int_{-\infty}^{\infty}|u(x)|^{2} d x,
\end{aligned}
$$

where $u(x)$ is the wave amplitude normalized on the intensity of the field at the nonlinear layer interface. Energy flow for the vacuum half space is found in the form $\int_{-\infty}^{0}|u(x)|^{2} d x=$ $\left[2 \operatorname{Im}\left(k_{1 x}\right)\right]^{-1}$. Integration over the periodic structure can be expressed as a sum over periods of the structure. If we apply Bloch's theorem for the Poynting vector we get $\int_{0}^{\infty}|u(x)|^{2}=$ $\mathcal{I}_{l} /\{1-\exp [-2 \operatorname{Im}(K) D]\}$. The integral over the period $\mathcal{I}_{\text {, }}$ can be calculated numerically using the transfer-matrix method.

Now we can evaluate the energy flow per unit length $P$ as the function of the frequency $\omega$ and propagation constant $\beta$. In our calculations, we consider the structure with the period $D=62.5 \mathrm{~nm}$, where the dielectric layer is twice as thick as the metal one. Metal-dielectric structures can operate in several different regimes: isotropic, anisotropic, or mixed [18]. We chose the parameters in such a way that our structure exhibits all possible regimes as we change the frequency. Dielectric is defined by the constant permittivity $\varepsilon_{d}=4$, and the permittivity of metal is described within the Drude model as $\varepsilon_{m}=1-\omega_{p}^{2} / \omega^{2}$ with plasma frequency $\omega_{p}=10 \mathrm{eV}$. The width of the nonlinear layer is set to $2 \mathrm{~nm}$ and its linear dielectric permittivity to unity. We consider two cases where the structure is terminated with either dielectric or metal layer, respectively. Figure 2 shows the dependence of the mode $P$ vs propagation constant $\beta$ for different frequencies in the case of the TE polarization. We can see that there exist no linear modes for low frequencies. The linear surface state [see Fig. 2(d)] is formed by an array of coupled dielectric waveguide modes in the metamaterial, and as any asymmetric waveguide mode it has a cutoff frequency. The nonlinear modes which do not originate from linear Tamm states have a finite energy flow threshold. The presence of the power threshold for the nonlinear waves was observed for a range of waveguiding structures [5-11]. We would like to mention that in more exotic waveguides, where the energy flows in different parts of the structure in opposite directions, there may exist nonlinear modes without power flow threshold [19], but having some minimum required total-energy circulation. In the shaded regions of Figs. 2(a) and 2(b) the modes are delocalized either in air or in the periodic structure, and the integral of the Poynting vector diverges. In addition, the derivative of the energy flow can change sign. This can potentially be an indication of the modulation instability of the surface states along the $y$ direction. Profiles of the electric field of the surfaces states are depicted in Figs. 2(c) and 2(d).

\section{B. TM polarized modes}

Most of the previous studies of the nonlinear surface waves dealt with TE polarized waves only. Here we would like to also study the properties of the TM polarized states, which have two components of the electric field. Due to this wave 

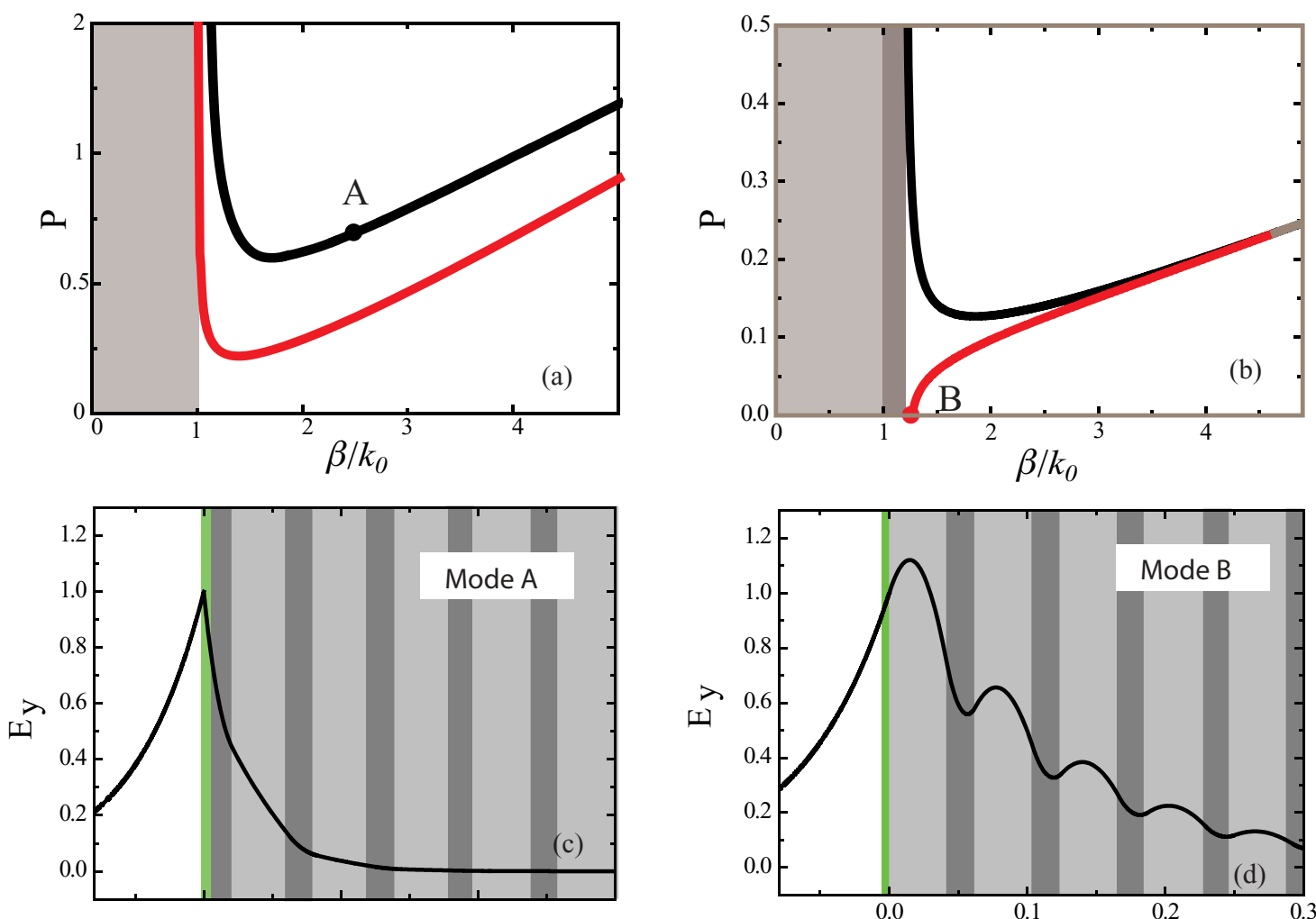

$x(\mu m)$

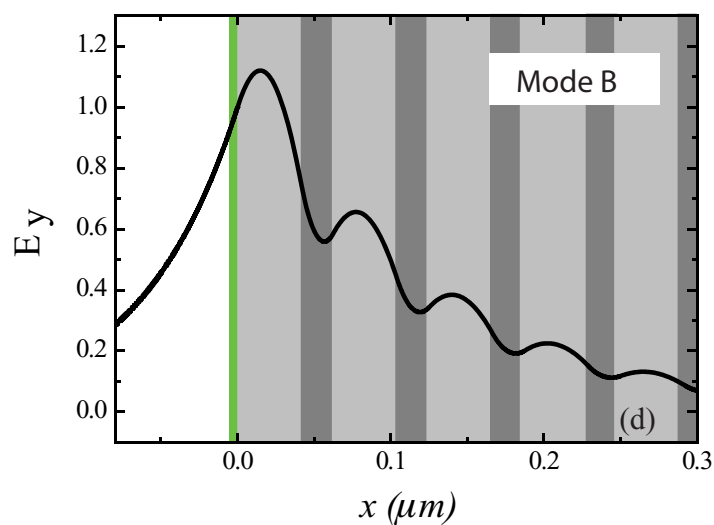

FIG. 2. (Color online) (a),(b) Energy flow vs propagation constant at the interface of metal-dielectric metamaterial in the case of TE polarization. Red (grey) lines correspond to the case when a dielectric layer terminates the structure, black lines correspond to the case when the structure is terminated at a metal layer. Frequency: (a) $2 \mathrm{eV}$, (b) $4 \mathrm{eV}$. Shaded rectangles correspond to the areas where the propagating solutions exist either in metamaterial or in vacuum. (c),(d) Characteristic profiles of the electric-field amplitude for the parameters corresponding to the points $\mathrm{A}$ and $\mathrm{B}$ in (a) and (b), respectively.

structure, the nonlinear dielectric permittivity now will now depend on two components of the electric field:

$$
\varepsilon=\varepsilon_{\text {lin }}+\chi_{3}\left(\left|E_{x}\right|^{2}+\left|E_{z}\right|^{2}\right) .
$$

We then write down the Maxwell's equations,

$E_{z}=-\frac{i}{k_{0} \varepsilon} \frac{\partial H_{y}}{\partial x}, \quad E_{x}=-\frac{\beta}{k_{0} \varepsilon} H_{y}, \quad \frac{\partial E_{z}}{\partial x}=i \beta E_{x}+i k_{0} \varepsilon H_{y}$.

Nonlinear differential equation for the field amplitude $H_{y}$ reads

$$
\frac{\partial^{2} H_{y}}{\partial x^{2}}-\frac{1}{\varepsilon} \frac{\partial \varepsilon}{\partial x} \frac{\partial H_{y}}{\partial x}+\left(\varepsilon k_{0}^{2}-\beta^{2}\right) H_{y}=0 .
$$

Assuming weak nonlinearity and negligibly small thickness of the nonlinear layer, we assume the dielectric permittivity to be constant across the layer. In this case the second term in Eq. (11) vanishes, and we obtain the solution for the field amplitude in the thin layer:

$$
H_{y}=A \sin [q(x+d)]+B \cos [q(x+d)],
$$

where $q$ and $d$ are defined similar to the TE case. Applying boundary conditions, we use the surface impedance $Z_{T M}=$ $E_{z} / H_{y}$ which reads

$$
Z_{T M}=\frac{T_{12}^{T M}}{e^{i K D}-T_{11}^{T M}} .
$$

The resulting dispersion equation then takes the form

$$
\left(\frac{\kappa}{k_{0}}-i Z_{T M}\right)=k_{0} d\left(1-\frac{\beta^{2}}{\varepsilon k_{0}^{2}}+i Z_{T M} \frac{\varepsilon \kappa}{k_{0}}\right),
$$

and

$$
\varepsilon-\varepsilon_{\text {lin }}=\chi_{3}\left|H_{y}\right|^{2}\left(\frac{\beta^{2}}{\varepsilon^{2}}+\kappa^{2}\right) .
$$

We notice that, contrary to the TE case, Eq. (14) is quadratic with respect to $\varepsilon$. It means that for each value of frequency and propagation constant, we have two possible values of $\varepsilon$ which satisfy the dispersion relation. We can illustrate this property for the example of a nonlinear layer at the boundary of a metaldielectric interface. In this case, one of the solutions always corresponds to the dielectric permittivity while the other corresponds to the permittivity of metal - in this case we would have the conventional surface plasmon at the interface. However, if we evaluate the relative difference between the roots of Eq. (14), $\varepsilon_{1}, \varepsilon_{2}, \delta=\left(\varepsilon_{1}-\varepsilon_{2}\right) /\left(\varepsilon_{1}+\varepsilon_{2}\right)$, we obtain

$$
\delta=\left(1-\frac{4 Z d^{2} \beta^{2} \sqrt{\beta^{2} / k_{0}^{2}-1}}{\sqrt{\beta^{2} / k_{0}^{2}-1}+Z-k_{0} d}\right)^{1 / 2} .
$$

Due to the fact that $d$ is small, the second term under the square root is always small, and thus the difference is always close to 
unity. Thus the difference of two roots is always of the order of the average of two roots. Thus if one of the roots satisfies the implied condition of weak nonlinearity, $\delta \varepsilon_{\mathrm{nl}} / \varepsilon \ll 1$, then the second root would definitely violate the condition. In what follows, we choose the root that meets the requirement of
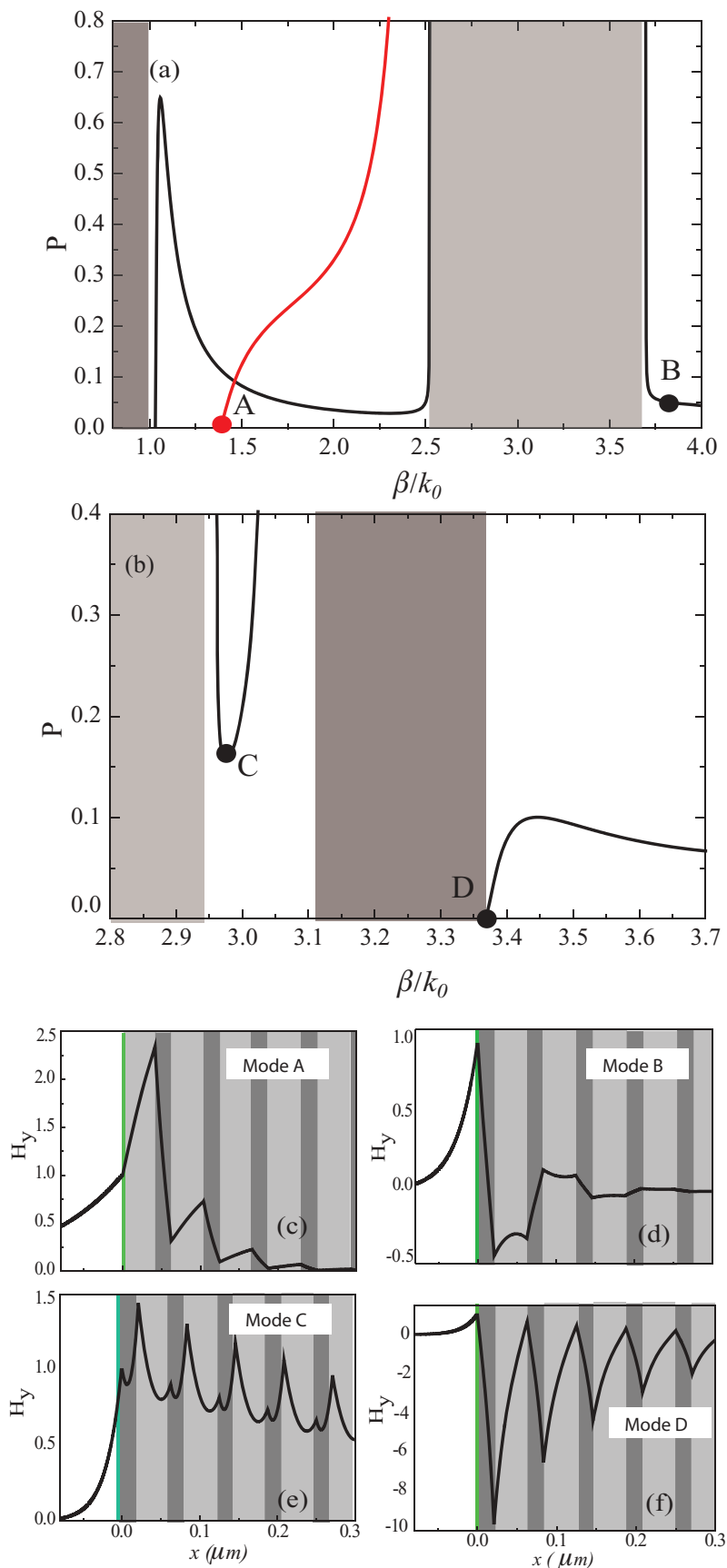

FIG. 3. (Color online) (a),(b) Energy flow vs propagation constant at the interface of metal-dielectric metamaterial in the case of TM polarization. Red (grey) lines correspond to the case when dielectric layer terminates the structure, black lines correspond to the case when the structure is terminated on the metal layer. Frequencies: (a) $2 \mathrm{eV}$, (b) $4 \mathrm{eV}$. Shaded rectangles correspond to the areas where the propagating solutions exist either in metamaterial or in vacuum. (c)-(f) Profiles of the electric-field amplitude for the values depicted with points A-D in (a) and (b), respectively. the weak nonlinearity, i.e., the closest to the linear dielectric permittivity of the nonlinear layer.

The calculation of the energy flow per unit length $P$ for the case of TM polarization is analogous to the case of TE polarization:

$$
\begin{aligned}
P & =\int_{-\infty}^{\infty} d x S_{z}=-\frac{1}{2} \int_{-\infty}^{\infty} \operatorname{Re}\left(E_{x} H_{y}^{*}\right) d x \\
& =\frac{\beta}{2 k_{0}}\left|E_{y}(0)\right|^{2} \int_{-\infty}^{\infty} \frac{1}{\varepsilon(x)}|u(x)|^{2} d x
\end{aligned}
$$

Energy flow vs propagation constant for the case of TM polarization is depicted in Figs. 3(a) and 3(b). We notice that for the case of TM polarization there are additional shaded regions corresponding to the propagation solutions in metal-dielectric layered structure. These propagating solutions are coupled surface plasmon polaritons of the individual metal-dielectric interfaces. The existence region for the propagating linear Bloch modes can be found from the inequality $|\cos (K D)|=\frac{1}{2}\left|\left(T_{11}+T_{22}\right)\right|<1$. A detailed analysis of these modes can be found in Ref. [18].

Profiles of the magnetic field for the surface states are shown in Figs. 3(c)-3(f). We notice that for the case of TM polarization some surface states look like coupled surface
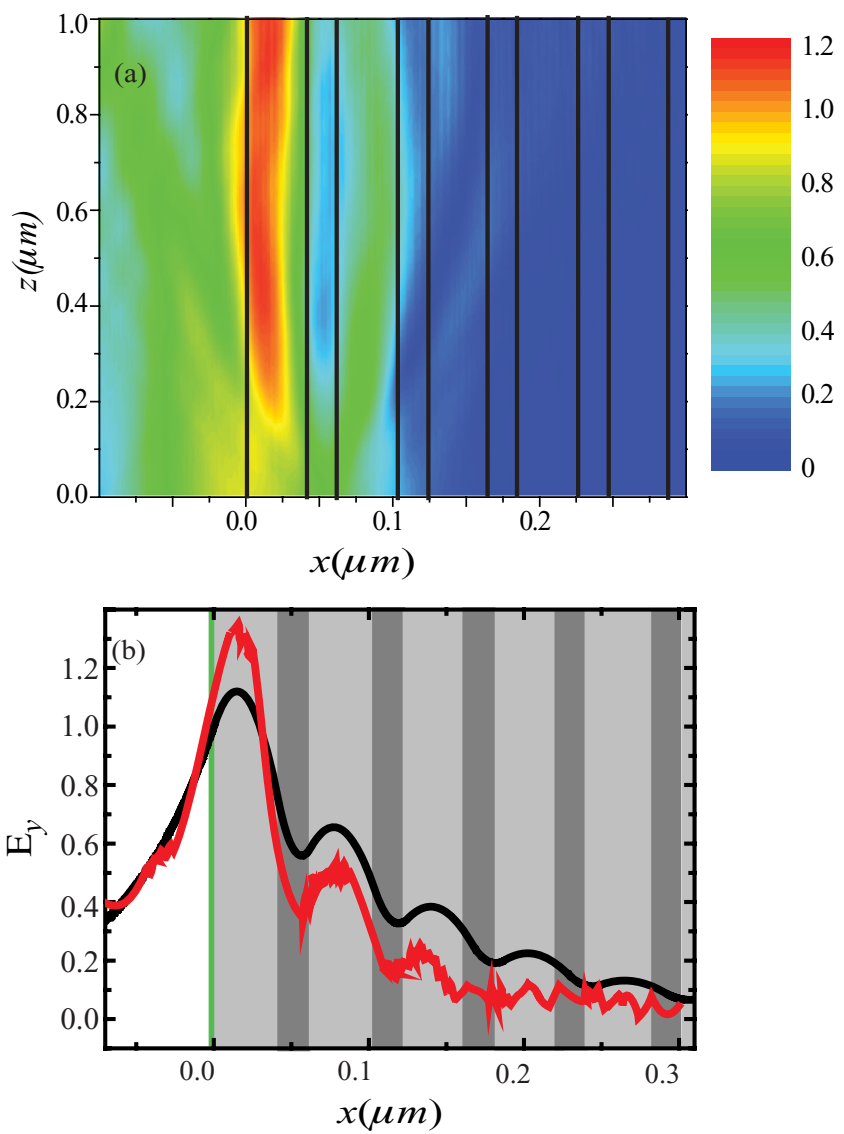

FIG. 4. (Color online) (a) Field map of electric-field distribution for the case of linear state depicted in Fig. 2. (b) Comparison of the numerical result for the field profile at the propagation length $2 \mu \mathrm{m}$ [red (grey) lines] and the analytical stationary results (black lines). Excitation frequency is $4 \mathrm{eV}$. 
plasmons at individual interfaces. We also observe that for the case of $\omega=4 \mathrm{eV}$ there exist neither linear nor nonlinear states in the structure with dielectric next to the nonlinear layer.

We should mention that limitations of the approach caused by the assumption of the thin layer and weak nonlinearity make us consider only those nonlinear surface states which appear due to the effective nonlinear change of the impedance of
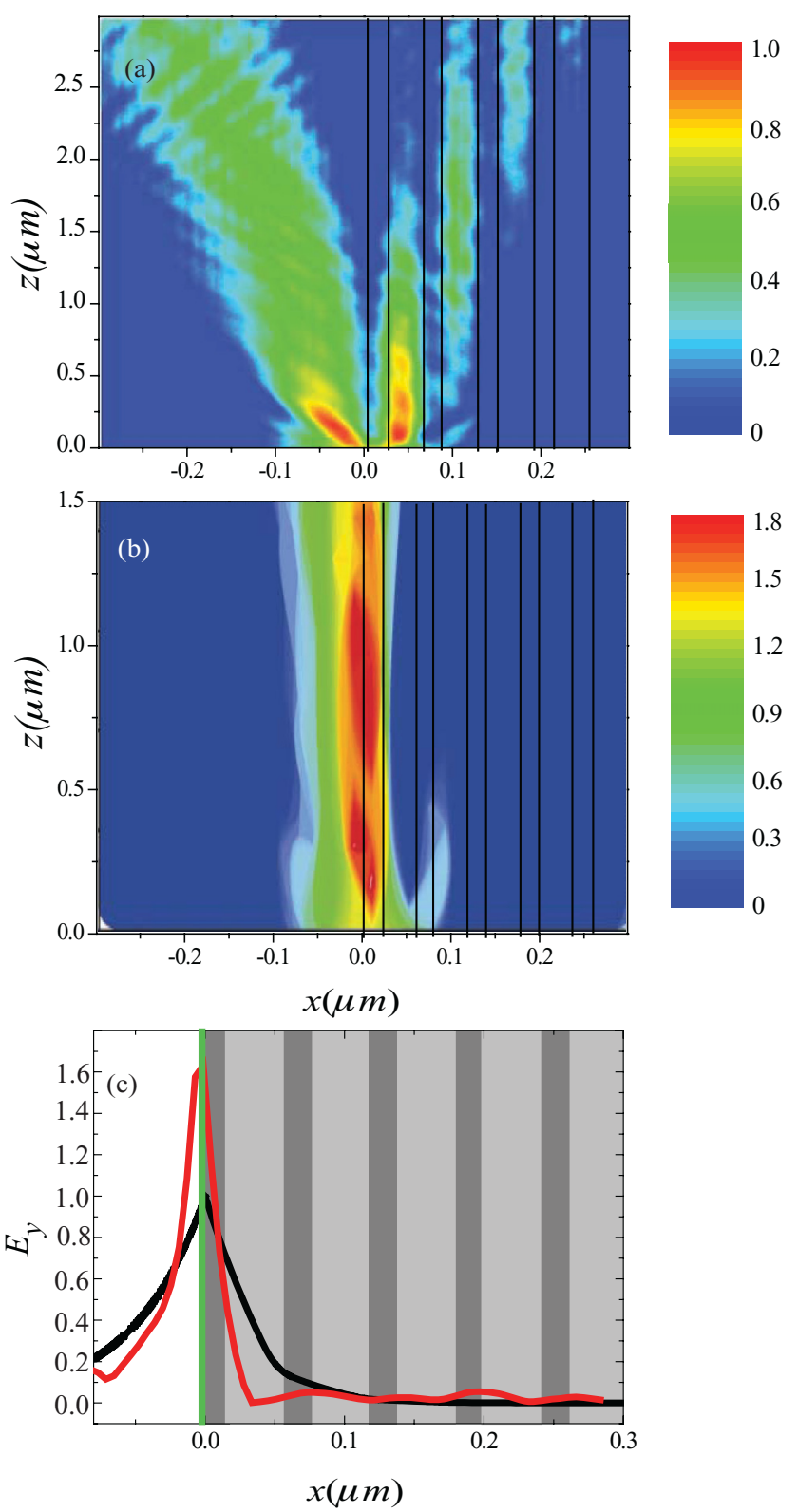

FIG. 5. (Color online) (a),(b) Field maps of electric-field distribution for the case of nonlinear surface-state excitation. (a) The energy is below the threshold energy and we observe the diffraction of the beam both in vacuum and in metamaterial. (b) The beam energy equal to the threshold energy. In this case the beam localizes at the interface forming a nonlinear surface state. (c) Comparison of numerical results for the field profile $z=2 \mu \mathrm{m}$ [red (grey) lines] and analytical stationary results (black lines). Excitation frequency is $2 \mathrm{eV}$. layered structure. This approach does not allow us to consider the nonlinear states associated with nonlinear guided waves of the thin nonlinear layer.

\section{GENERATION OF SURFACE MODES}

To verify our analytical results, we perform the numerical simulations using the Beam propagation method (BPM) [20]. We consider the case of the TE polarization which is described with the Helmholtz equation for the electric field,

$$
\frac{\partial^{2} E}{\partial x^{2}}+\frac{\partial^{2} E}{\partial z^{2}}+k_{0}^{2} \varepsilon(x) E=0,
$$

where $\varepsilon$ includes both linear and nonlinear parts. We then apply the paraxial approximation, and express the field amplitude in the form $E=A(x, z) e^{i \beta z}$, where $A$ changes slowly along the propagation direction, i.e., $\left|\partial^{2} A / \partial z^{2}\right| \ll 2 \beta|\partial A / \partial z|$. If we then substitute this expression into the Helmholtz equation (18) and introduce new dimensionless variables $\bar{x}=\beta x, \bar{z}=\beta z$, we obtain the familiar nonlinear Schroedinger equation:

$$
2 i \frac{\partial A}{\partial \bar{z}}=-\frac{\partial^{2} A}{\partial \bar{x}^{2}}+\left(1-\varepsilon_{\operatorname{lin}}(\bar{x}) \frac{k_{0}^{2}}{\beta^{2}}\right) A-\frac{k_{0}^{2}}{\beta^{2}} S(\bar{x})|A|^{2} A,
$$

where

$$
S(x)=\left\{\begin{array}{l}
1,0<x / \beta<d \\
0, x / \beta>d, x / \beta<0 .
\end{array}\right.
$$

Equation (19) is solved numerically using the CranckNikolson scheme. We use the absorbing boundary conditions and Gaussian beam with the center at the nonlinear layer as the initial condition at $z=0$.

Figure 4(a) shows the results of our numerical simulations for the linear case, the corresponding mode is depicted in Fig. 2(b) with marker B. We observe that the input Gaussian beam generates a linear surface mode as it propagates along the interface. The profile of the electric field after the certain propagation distance becomes close to the stationary mode is

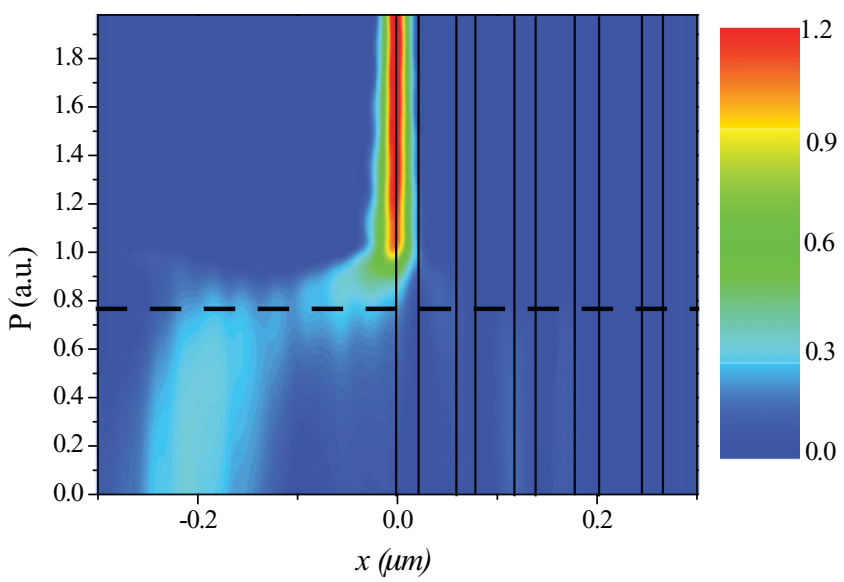

FIG. 6. (Color online) Field map of the electric-field distribution after $2-\mu \mathrm{m}$ propagation distance for the frequency $2 \mathrm{eV}$ and $\beta=$ $2.5 k_{0}$. Horizontal dashed line shows the threshold power predicted with the analytical theory. 
described by the analytical theory [shown in Fig. 4(b) by a black curve].

Figures 5(a) and 5(b) show the propagation of the input Gaussian beam launched near the nonlinear interface; the corresponding mode is depicted in Fig. 2(a) (A). Figure 5(a) corresponds to the case when there is insufficient power to excite a nonlinear mode, and the wave is diffracted. When the power of the input beam reaches the threshold [see Fig. 5(b)], the field localizes at the interface forming the nonlinear surface state with the profile close to one obtained from the analytical theory [shown in Fig. 5(c) by a black solid curve].

To compare the power threshold values obtained from the analytical and numerical calculations, in Fig. 6 we plot the distribution of the electric field after $2 \mu \mathrm{m}$ propagation versus input energy flow. Each horizontal cross section of this figure corresponds to the electric-field profile for a given input power of the Gaussian beam. We notice that when the input power reaches a threshold value, the field localizes at the interface. The threshold pump power obtained numerically is slightly larger than that found analytically [shown by dashed horizontal line on Fig. 6]. This can be explained by the fact that the Gaussian initial beam profile used in numerical simulations does not coincide with the stationary surface wave profile, and a part of the input energy is diffracted from the beam when it transforms into the surface state.

\section{CONCLUSIONS}

We have presented a simplified analytical approach for the calculation of nonlinear dispersion of surface states in metaldielectric nanostructures. We have shown that along with linear surface states, such terminated structures support nonlinear states having a finite threshold power. We have shown that, for the case of TM polarization two types of localized modes may exist even for the wavelengths much larger than the structure period. We have revealed that for the case of TM polarization up to four modes having the same frequency can be supported by the structure, i.e., the TM modes demonstrate multiple stability.

\section{ACKNOWLEDGMENTS}

This work was supported by the Ministry of Education and Science of the Russian Federation, RFBR and Dynasty Foundation (Russia), EPSRC (UK), and the Australian Research Council.
[1] P. Yeh, A. Yariv, and A. Y. Cho, Appl. Phys. Lett. 32, 104 (1978).

[2] D. Mihalache, D. Mazilu, and F. Lederer, Opt. Commun. 59, 391 (1986).

[3] F. Lederer and D. Mihalache, Solid State Commun. 59, 151 (1986).

[4] D. Mihalache, M. Bertolotti, and C. Sibilia, Prog. Opt. 27, 229 (1989).

[5] K. G. Makris, S. Suntsov, D. N. Christodoulides, G. I. Stegeman, and A. Hache, Opt. Lett. 30, 2466 (2005).

[6] M. Molina, R. Vicencio, and Yu. S. Kivshar, Opt. Lett. 31, 1693 (2006).

[7] Y. V. Kartashov, L. Torner, and V. A. Vysloukh, Phys. Rev. Lett. 96, 073901 (2006).

[8] S. Suntsov, K. G. Makris, D. N. Christodoulides, G. I. Stegeman, A. Hache, R. Morandotti, H. Yang, G. Salamo, and M. Sorel, Phys. Rev. Lett. 96, 063901 (2006).

[9] G. A. Siviloglou, K. G. Makris, R. Iwanow, R. Schiek, D. N. Christodoulides, G. I. Stegeman, Y. Ming, and W. Sohler, Opt. Express 14, 5508 (2006).
[10] C. R. Rosberg, D. N. Neshev, W. Krolikowski, A. Mitchell, R. A. Vicencio, M. I. Molina, and Yu. S. Kivshar, Phys. Rev. Lett. 97, 083901 (2006).

[11] E. Smirnov, M. Stepic, C. E. Ruter, D. Kip, and V. Shandarov, Opt. Lett. 31, 2338 (2006).

[12] I. Iorsh, P. A. Belov, I. V. Shadrivov, and Yu. S. Kivshar, Phys. Status Solidi: Rapid R. Lett. 6, 43 (2012).

[13] Yu. S. Kivshar, Laser Phys. Lett. 5, 703 (2008).

[14] S. M. Vukovic, I. V. Shadrivov, and Yu. S. Kivshar, Appl. Phys. Lett. 95, 041902 (2009).

[15] S. H. Nam, E. Ulin-Avila, G. Bartal, and X. Zhang, Opt. Lett. 35, 1847 (2010).

[16] S. H. Nam, E. Ulin-Avila, G. Bartal, and X. Zhang, Opt. Express 18, 25627 (2010).

[17] A. Karlsson, IEEE Trans. Antennas Propag. 57, 144 (2009).

[18] A. A. Orlov, P. M. Voroshilov, P. A. Belov, and Yu. S. Kivshar, Phys. Rev. B 84, 045424 (2011).

[19] I. V. Shadrivov, A. A. Sukhorukov, Yu. S. Kivshar, A. A. Zharov, A. D. Boardman, and P. Egan, Phys. Rev. E 69, 016617 (2004).

[20] G. Agrawal, Nonlinear Fiber Optics (Academic, New York, 2001). 